\title{
OpINION
}

\section{CanMEDS and the combat against antibiotic resistance}

\author{
Timothy S.H. Kwok \\ Class of 2018, Faculty of Medicine, University of Ottawa
}

$\mathrm{R}$ ecently, I finished an Internal Medicine elective at The Ottawa Hospital. There, I was privileged to care for some of the city's sickest patients. As the elective progressed, I began to notice a pattern. Every time medical staff entered a patient's room, they would frantically put on gowns, facemasks, and gloves covering every part of their bodies. Curious, I wondered why? Suddenly, out of the corner of my eye, I noticed a sea of signs labeled "MRSA Protocols." That evening, I reflected on how the problem of "superbugs" had gotten so out of hand. In lectures, we had learned the genetic reasons behind the development of antibiotic resistance but I realized there must be reasons beyond the realm of biology. In fact, there is an interplay of economic, psychosocial, and political factors that also contribute to society's mishandling of such an invaluable resource.

As medical students, we are introduced to the CanMEDS framework at an early point in our training. CanMEDS was created by The Royal College of Physicians and Surgeons of Canada in the 1990s to promote competencies in seven key pillars for physicians in training to improve patient care (Figure 1). I pondered deeply at how the seven roles behind this prominent medical education dogma could play a part in combating the economic, psychosocial, and political factors of antibiotic resistance.

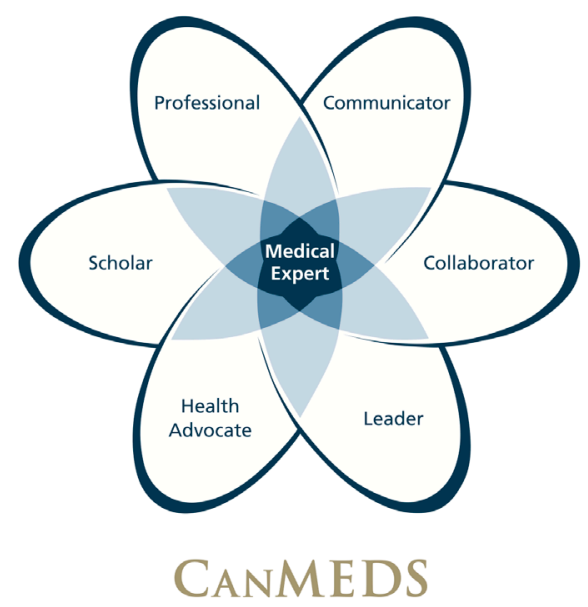

Figure I. CanMEDS 2015 Framework displaying the seven cornerstone roles. Copyright (C) 2015 The Royal College of Physicians and Surgeons of Canada. http://rcpsc.medical.org/- canmeds.

Reproduced with permission.

At present, there are 17 antibiotic-resistant microorganisms in the United States that result in roughly 23,000 deaths annually. ${ }^{1}$ To understand the current state of antimicrobial resistance, a historical perspective must be considered. The first two antibiotics developed for human use were penicillin and the sulfonamides in 1928 and 1937 respectively. $^{2}$ Within several years, resistance had developed against both. $^{2}$ To date, resistance has developed in every antibiotic class, most often within several years of its introduction to market. ${ }^{2}$ Scientists have theorized multiple explanations behind this phenomenon, boiling it down to an incessant biological arms race between humans and bacteria. Whenever a bacterial strain develops resistance, humans counter with another class of antibiotics. This malicious cycle of antibiotic use and gene exchange culminates in strains of bacteria that ultimately become unyielding to all anti-microbial weapons humans have to offer. Regardless of the biological mechanisms, this raises the question as to why we have run out of bactericidal machinery to continue this arms race. Like many issues in contemporary medicine, economics plays a pivotal role. It is estimated that bringing a new drug from bench to bedside takes a decade and costs around 2.6 billion dollars. ${ }^{3}$ Furthermore, current Canadian patent laws allow for drug exclusivity in the market for only 20 years from the commencement of research and development. ${ }^{4}$ Considering the massive costs and time commitment to develop antibiotics, drug companies find it difficult to reap the economic benefits, since bacteria are likely to become resistant to the antibiotic in a few years. As a case in point, only two classes of novel antibiotics, fidaxomicin and diarylquinolines, have been introduced to market during the past 10 years. ${ }^{5}$ To make matters worse, even the old generic antibiotics are frequently short in supply, forcing physicians to use broader and less optimal drugs. ${ }^{6}$ Moreover, antibiotics are mostly tailored for acute usage. If a patient takes a week-long course of antibiotics and is cured from the illness, then pharmaceutical companies cannot profit from the medication as they would if the condition were chronic. In summary, it is understandable from a financial standpoint why antibiotic development has become so stagnant in recent years.

The main issue at hand is how we, as future healthcare leaders, can combat this catastrophe. To that notion, we all need to play the role of Health Advocates and steer for change. It is clear that the biological mechanisms behind antibiotic resistance are unyielding. What can change is our ability to 
make antibiotic development more lucrative to pharmaceutical companies, thus creating economic incentives to pave the way for new drug development. By reaching out to our local Members of Parliament and the Ministry of Health with lobbying days for increased research grants, and by fundraising for antibiotic research, every medical student can play a role. In fact, results of lobbying efforts have met some success in the United States, culminating in a proposed bill to expedite drug approvals that target "a serious or life-threatening disease, condition or indication".

Another role that we could assume is that of a Scholar. To the general public, a career in research seems neither exciting nor glamorous. By shining the spotlight on medical research in medical curriculums around Canada, hopefully many more students will be attracted to a career in research. As our academic workforce ages, there will be an increasing need for new blood to be infused into the knowledge sector, leading the way for antibiotic discoveries in the future.

To address the issue of antibiotic supply shortage the supply chain for antibiotics in Canada can be strengthened by improving communication between prescribers and pharmaceutical companies. This could be acomplished by using electronic records and implementing a mandatory one-year notice period before drug production facilities can cease operations. ${ }^{6}$

I recall visiting a clinic last winter complaining of a sore throat and a cough. After seeing the doctor, I left the clinic with a prescription for an antibiotic. Looking back, I pondered whether I really did need the medication. It is known amongst healthcare workers that antibiotics are useless against the common cold. Yet it is estimated that roughly $60 \%$ of the time doctors still prescribe antibiotics, albeit inappropriately, which is one of the most important drivers of resistance. ${ }^{8}$ To analyze the rationale behind this, we must look at physician culture and more globally, the psychosocial factors behind antibiotic resistance. Constantly faced with the immense pressure of clinical decisions determining life or death, doctors are primed to "better be safe than sorry" and, consequently, tend to conservatively overprescribe. To improve this situation, I firmly believe in the implementation of even more rigorous continuing education for medical students, residents, and physicians. I am a proponent of the antimicrobial stewardship program at major hospitals, whereby a dedicated team of infectious disease specialists and pharmacists track antibiotic usage and provide ad hoc analysis regarding its appropriateness to educate prescribers. However, I would like to see this program implemented nation-wide, especially in community settings.
In fact, we can turn to examples where antibiotic stewardship programs were implemented and met with resounding success. One of the most prominent examples is the state of California, which mandated antimicrobial stewardship legislation in $2014 .{ }^{9}$ In Canada, antimicrobial stewardship is labeled as a Required Organizational Practice by Accreditation Canada, the regulatory body that accredits Canadian hospitals. ${ }^{10}$ This not only fosters an environment of Collaboration between clinicians, laboratory doctors, nursing staff, pharmacists and hospital administrators, but also ensures that all parties become effective Communicators, striving for the goal of antibiotic stewardship. By honing the Medical Expert and Leader roles, doctors can competently practice evidence-based medicine to correctly make clinical decisions benefiting patient safety, while safeguarding the limited resources that our healthcare system possesses.

Another side of the problem rests with the patient. For many patients, if they leave the doctor's office with no medication or follow-up, they feel as if the appointment was a waste of time. Nevertheless, with the common cold, supportive therapy is actually the best treatment. Thus, it is important for physicians to assume the roles of an educator and Communicator to advise patients on basic mechanisms behind the common cold and consequently, the rationale behind not utilizing an antibiotic. Physicians, as Professionals, are undoubtedly the guardians of the healthcare system and have the ethical duty of allocating resources appropriately as well as engaging in social accountability, promotion of public good, and commitment to patient safety. The use of antibiotics is no exception, and proper education both for doctors and patients can go a long way in managing our precious assets.

On a policy level, governmental factors are contributing to antibiotic resistance, stemming from the Parliament's inability to balance the interests of corporations and the public. These competing interests are most apparent in the agricultural industry, where widespread usage of antibiotics for the purpose of "preventing disease" in livestock accounts for 50\% of Canada's total antimicrobial use. ${ }^{11}$ The industry standard of prophylactically enhancing animal feeds with antibiotics is seeding a fertile ground for resistance. In developing solutions, we should analyze the successes of other countries, with perhaps the most outstanding example being Denmark. Their ability to apply science to policy spearheaded the shift toward a prophylactic antibiotic-free livestock industry. There is evidence suggesting a correlation between antibiotic use in agriculture and poor human health outcomes. ${ }^{12}$ In contrast, Danish curtailment of antibiotics in livestock has actually posed no detriment to the pork industry. ${ }^{12}$ Although Danish antibiotic 
use for pigs dropped by more than $50 \%$ from 1992 to 2008, production increased by 19 million pigs during that same time span. ${ }^{13}$ These results can serve notice to agricultural companies that there is indeed an added financial benefit to discontinuing use of prophylactic antibiotics in livestock. Nonetheless, our fundamental shortcoming is the Canadian government's reluctance to act upon such evidence. We, as future physicians, should promote the role of Health Advocate in convincing politicians to enact laws to protect antibiotic resources. Ultimately, in a democratic country such as Canada, it is the public that drives political actions. This is why we need to elucidate the role agriculture has on antibiotic depletion to the masses, so that they may join our mission in enacting political change. By holding lobby days, media campaigns, and rooting ourselves in the political scene, we can gain more influence in improving our societies health.

In conclusion, the plight of our antibiotic resources can be attributed to economic, psychosocial, and political reasons in addition to biological factors. Notwithstanding these issues, it is even more important to pinpoint areas of improvement to ameliorate this situation. As future physicians, we have the obligation to assume the CanMEDS roles of Scholar, Communicator, Collaborator, Professional, Health Advocate, and Leader to become true Medical Experts. By doing so, we not only conserve one of the greatest miracles of modern medicine but also enhance the health of Canadians across the country.

\section{References}

1. Centers for Disease Control and Prevention. Antibiotic Resistance Threats in the United States, 2013. Atlanta: United States Department of Health and Human Services 2013;11-17.

2. Davies J, Davies D. Origins and evolution of antibiotic resistance. Microbiol Mol Biol Rev 2010;74(3):417-33.

3. Lindsley CW. New statistics on the cost of new drug development and the trouble with CNS drugs. ACS Chem Neurosci 2014;5(12):1142.

4. Canadian Patent Act. R.S., 1985, c. P-4, s. 1

5. Lewis K. Platforms for antibiotic discovery. Nat Rev Drug Discov 2013;12(5):371-87.

6. Lynas K. Quebec pharmacists and physicians call for legislative changes to combat drug shortages in Canada. Can Pharm J 2012;145(3):106.

7. Promise for Antibiotic and Therapeutics for Health Act, S. 185, 114th Cong., 1st Sess. (2015).

8. Barnett ML, Linder J. Antibiotic prescribing to adults with sore throat in the United States, 1997-2010. JAMA Intern Med 2014;174(1):138-40.

9. California Department of Public Health. "The California Antimicrobial Stewardship Program Initiative" 16 June 2016. $<$ http://www.cdph.ca.gov/programs/hai/Pages/antimicrobialStewardshipProgramInitiative.aspx> (19 June 2016).

10. Accreditation Canada. "Antimicrobial Stewardship ROP" 20 September 2012. <https://accreditation.ca/antimicrobial-stewardship-rop> (19 June 2016).

11. Van Gerwen J. "Antimicrobial Resistance in Agriculture" 17 June 2014. <http://www.omafra.gov.on.ca/english/livestock/ animalcare/amr/facts/13-077.htm> (28 April 2015).

12. Levy S. Reduced antibiotic use in livestock: how Denmark tackled resistance. Environ Health Perspect 2014;122(6):A1605 .

13. Aarestrup FM, Jensen VF, Emborg HD, Jacobsen E, Wegener HC. Changes in the use of antimicrobials and the effects on productivity of swine farms in Denmark. Am J Vet Res 2010;71(7):726-33. 